\title{
Evaluación de accesiones cubanas de papaya (Carica papaya L.) ante la mancha anular
}

\author{
Douglas Rodríguez ${ }^{1,3}$, Maruchy Alonso², Yoel Tornet ${ }^{1}$, Lázaro Valero ${ }^{1}$, Emi Rainildes Lorenzetti ${ }^{3}$ y Romualdo Pérez ${ }^{1}$. \\ ${ }^{1}$ Unidad Científico-Tecnológica de Base de Jaguey Grande-Instituto de Investigaciones en Fruticultura Tropical. Calle 24 No. 1702, Torriente, Matanzas. \\ Cuba. E-mail:rodriguezdouglas1975@yahoo.com.br. ${ }^{2}$ Departamento de Mejoramiento Genético-Instituto de Investigaciones en Fruticultura Tropical. \\ Ave: 7ma e/ 30 y 32, Miramar, Playa, Ciudad Habana. Cuba. ${ }^{3}$ Universidad Federal de Lavras, Departamento de Fitopatologia, Lavras-MG, Brasil. \\ Autor para correspondência: Emi Rainildes Lorenzetti (elorenzetti@gmail.com)
}

Data de chegada: 10/10/2010. Aceito para publicação em: 11/01/2013.

\section{RESUMEM}

Rodríguez, D.; Alonso, M.; Tornet; Y.; Valero, L.; Lorenzetti, E.R.; Castillo, R.. Evaluación de accesiones cubanas de papaya (Carica papaya L.) ante la mancha anular. Summa Phytopathologica, v.39, n.1, p.24-27, 2013.

Se colectaron en diferentes regiones de Cuba semillas de las accesiones de papayo Tallo Morado de Nava, Amarilla de Duaba, Amarilla de Nava y Sapote de Pilón. Estas se plantaron en condiciones de campo en Jagüey Grande, Matanzas - Cuba, donde se evaluó la presencia e intensidad de síntomas de mancha anular, siguiendo una escala graduada del 1 al 5. Los resultados mostraron un incremento temporal de la proporción de plantas afectadas en todas las accesiones. La accesión Amarilla de Duaba mostró los síntomas a los dos meses después de la primera evaluación (MDPE), mientras que el resto lo hizo a los cinco MDPE, aunque con proporciones que variaron entre ellas. A los siete MDPE las accesiones Amarilla de
Duaba y Amarilla de Nava mostraron proporciones idénticas de plantas enfermas, mientras que Tallo morado de Nava y Sapote de Pilón mostraron proporciones inferiores. La intensidad de los síntomas se incrementó en los diferentes órganos siguiendo el siguiente orden: tallo $(1,7$ a 2,66$)$, pecíolos $(2,21$ a 3,03$)$ y follaje $(3,44-4,03)$. En los frutos la intensidad de los síntomas observados fue inferior a 2,5, considerados como leves de acuerdo a su intensidad y distribución. Se ofrecen los primeros resultados sobre la susceptibilidad a la mancha anular de estas accesiones, siendo de gran utilidad para los productores, así como para los programas de mejoramiento genético en el país.

Palabras clave: mejoramiento genético, susceptibilidad, intensidad de síntomas, virus.

\section{RESUMO}

Rodríguez, D.; Alonso, M.; Tornet; Y.; Valero, L.; Lorenzetti, E.R.; Castillo, R. Avaliação de acessos cubanos de mamão papaya (carica papaya L.) à mancha anelar. Summa Phytopathologica, v.39, n.1, p.24-27, 2013.

Os acessos de mamão Tallo morado de Nava, Amarilla de Duaba, Amarilla de Nava e Sapote de Pilón foram coletados em diferentes regiões de Cuba e plantados em condições de campo em Jagüey Grande, Matanzas - Cuba. Nestas plantas foram avaliadas a presença e intensidade dos sintomas de mancha anelar, usando uma escala graduada de 1-5. Os resultados mostraram um aumento, com o tempo, na proporção de plantas afetadas em todos os acessos. O acesso Amarilla de Duaba apresentou sintomas dois meses após a primeira avaliação (MDPE), enquanto para os restantes foram observados sintomas após cinco MDPE, contudo em proporções diferentes entre eles. Aos sete
MDPE os acessos Amarilla de Duaba e Amarilla de Nava, apresentaram proporções iguais de plantas doentes, enquanto Tallo morado de Nava e Sapote de Pilon apresentaram menores proporções. O aumento da intensidade dos sintomas foi diferente nos órgãos da planta na seguinte ordem: caule $(1,7-2,66)$, pecíolos $(2,21-3,03)$ e folhagens $(3,44-4,03)$. Nos frutos a intensidade dos sintomas observados foi inferior a 2,5, considerada leve em função da sua intensidade e distribuição. Estes são os primeiros resultados sobre a susceptibilidade a mancha anelar destes acessos, sendo muito úteis para os produtores, bem como para os programas de melhoramento do país.

Palavras-chave adicionais: melhoramento genético, suscetibilidade, intensidade de síntomas, vírus.

\section{ABSTRACT}

Rodríguez, D.; Alonso, M.; Tornet; Y.; Valero, L.; Lorenzetti, E.R.; Castillo, R. Assessment of Cuban papaya (Carica papaya L.) accessions against ringspot. Summa Phytopathologica, v.39, n.1, p.24-27, 2013.

The papaya accessions "Tallo morado de Nava", "Amarilla de Duaba", "Amarilla de Nava" and "Sapote de Pilón" were collected from different regions of Cuba and planted under field conditions in Jagüey Grande, Matanzas - Cuba. These plants were assessed for the presence and intensity of ringspot symptoms, using a graduated scale of 1-5. Results showed an increase, with time, in the proportion of affected plants for all accessions. The accession "Amarilla de Duaba" had symptoms two months after the first assessment (MAFA), while for the remaining accessions, symptoms were noted after only five MAFA but at proportions that differed among them. At seven MAFA, the accessions "Amarilla de Duaba" and "Amarilla de Nava" had proportions equal to those of diseased plants, while "Tallo morado de Nava" and "Sapote de Pilon" showed lower proportions. The increase in symptom intensity was different for the plant organs in the following order: stem (1.7-2.66), petioles (2.21-3.03) and leaves (3.44-4.03). For fruits, the intensity of the observed symptoms was inferior to 2.5 , considered light based on their intensity and distributions. These are the first results about ringspot susceptibility for these accessions, which are highly useful for farmers, as well as for breeding programs in the country.

Additional keywords: breeding programs, susceptibility, symptom intensity, virus 
La papaya (Carica papaya L.) es una importante fruta de la familia Caricaceae, con alto valor nutricional y económico, la cual es cultivada en diversas regiones tropicales y subtropicales del mundo. Es muy común en la América tropical, siendo muy apreciada principalmente para su consumo in natura (8).

El centro de origen de la papaya es la Bacia Amazónia Superior. No obstante, el Caribe constituye una zona de diversificación secundaria, donde las poblaciones locales presentan adaptaciones a las condiciones regionales (10).

Como muchos otros cultivos agrícolas, la papaya puede ser afectada por diferentes plagas y enfermedades que reducen la longevidad de las plantaciones, así como su producción y calidad de frutos. Dentro de las enfermedades se destacan las producidas por virus, provocando cuantiosos daños al cultivo $(14,16)$.

El Papaya ring-spot virus tipo P (PRSV-P) causante de la mancha anular de la papaya, constituye uno de los factores más limitante para la producción de esta fruta. PRSV-P infecta tanto papaya como cucurbitáceas y chenopodiaceas y se encuentra en la mayoría de las regiones productoras de papaya del mundo (21). Estas incluyen Haway, Filipinas, Taiwán, Tailandia, Vietnam, India, Sri Lanka, Australia, África, Florida, Brasil, Venezuela, México y las islas del Caribe, entre otros $(17,18,1,23,16)$.

PRSV-P es transmitido de forma no persistente por más de veinte especies de áfidos $(22,15)$ a la papaya y a una amplia gama de plantas hospedantes (12). Los síntomas en papayo consisten en engrosamiento y amarillamiento de las nervaduras de hojas jóvenes, moteado y/o mosaico foliar y en muchos casos ampollamiento severo y distorsión de las hojas. En los pecíolos y el tallo se desarrollan manchas de color verde olivo y aspecto aceitoso, mientras que los frutos verdes desarrollan en su superficie manchas o círculos concéntricos de color verde oscuro, que al madurar se pueden tornar anaranjado-parduzcos. El vigor de las plantas y la producción suelen afectarse en dependencia de la edad de la planta en el momento de la infección $(17,22)$.

El uso de variedades y cultivares tolerantes y/o resistentes constituye actualmente una de las alternativas más prometedoras para lograr un manejo efectivo de esta enfermedad, y con ello, altas producciones de esta fruta $(24,11,21)$.

En Cuba, donde la base genética de la papaya es estrecha, sustentada fundamentalmente en el cultivo de la variedad Maradol Roja, la diversificación de variedades y accesiones de papaya podría constituir una alternativa para la diversificación de la fruticultura en el país. Para lograr esto, se vienen desarrollando varios estudios sobre el comportamiento agroproductivo y fitosanitario de variedades introducidas al país $(2,3,4,20)$.

Durante varios años los productores cubanos han utilizado accesiones que mantienen elevadas producciones y bajos niveles de afectación por mancha anular. Estas accesiones pudieran constituir alternativas para la diversificación genética de la papaya, así como, fuentes promisorias para los programas de mejoramiento genético del papayo en el país. Por todo lo planteado anteriormente, este trabajo tuvo como objetivo proyectar y evaluar el comportamiento ante la mancha anular de cuatro accesiones de papaya en áreas de alta incidencia de PRSV-P.

\section{MATERIALES Y MÉTODOS}

El estudio se desarrolló en áreas experimentales del lote AG-41 pertenecientes a la Empresa de Cítricos Victoria de Girón, bajo las condiciones edafoclimáticas de Jagüey Grande, Matanzas - Cuba (16).
Para ello se evaluaron durante siete meses (mayo a noviembre de 2007), cuatro accesiones de papaya plantadas según un diseño de bloques al azar con tres réplicas y 10 plantas por réplica, a un marco de plantación de $2.0 \mathrm{~m}$ x $3.0 \mathrm{~m}$. Las accesiones evaluadas fueron: Tallo morado de Nava, Amarilla de Duaba, Amarilla de Nava y Sapote de Pilón. Todas ellas caracterizadas recientemente por la técnica de AFLP (10).

De cada una de las accesiones se muestrearon mensualmente todas las plantas. Se evaluó la presencia de síntomas típicos de la enfermedad de acuerdo a lo descrito por varios autores en tallo, pecíolos, follaje y frutos $(18,11)$. Para ello se elaboró la siguiente escala graduada: 0 ausencia de síntomas; 1 - sospecha de síntomas, pero sin estar bien definido; 2 - síntoma evidente, pero puntual en el órgano; 3 - síntoma que abarca hasta el $50 \%$ del órgano afectado y no se encuentra generalizado en todos los órganos del mismo tipo; 4 - síntoma severo, pero que sólo afecta entre el 51 y $75 \%$ del órgano y se encuentra más generalizado en los órganos; 5 - síntoma severo y generalizado en todos los órganos del mismo tipo (más del 75\%).

Con los datos obtenidos se determinó la proporción de plantas afectadas en el tiempo (se consideró que una planta estaba enferma cuando dos o más de sus órganos alcanzaron el grado tres en la escala empleada), comparando en cada evaluación las selecciones criollas mediante comparación de proporciones para p£0.05. Se determinó además el grado de síntomas por órgano; para lo cual se comprobó la normalidad y homogeneidad de la varianza. Posteriormente, en el análisis de varianza, las comparaciones entre las medias se realizó mediante el Test de Tuckey ( $\mathrm{p} £ 0.05$ ). Todos los análisis fueron realizados utilizando el paquete estadístico Statistica Versión 6.0 (Statsoft, Tulsa, AZ, USA).

\section{RESULTADOS Y DISCUSIÓN}

La Figura 1 muestra el incremento de la proporción de plantas afectadas para cada accesión de papayo en el tiempo. Amarilla de Duaba fue la primera en mostrar síntomas de la enfermedad con una

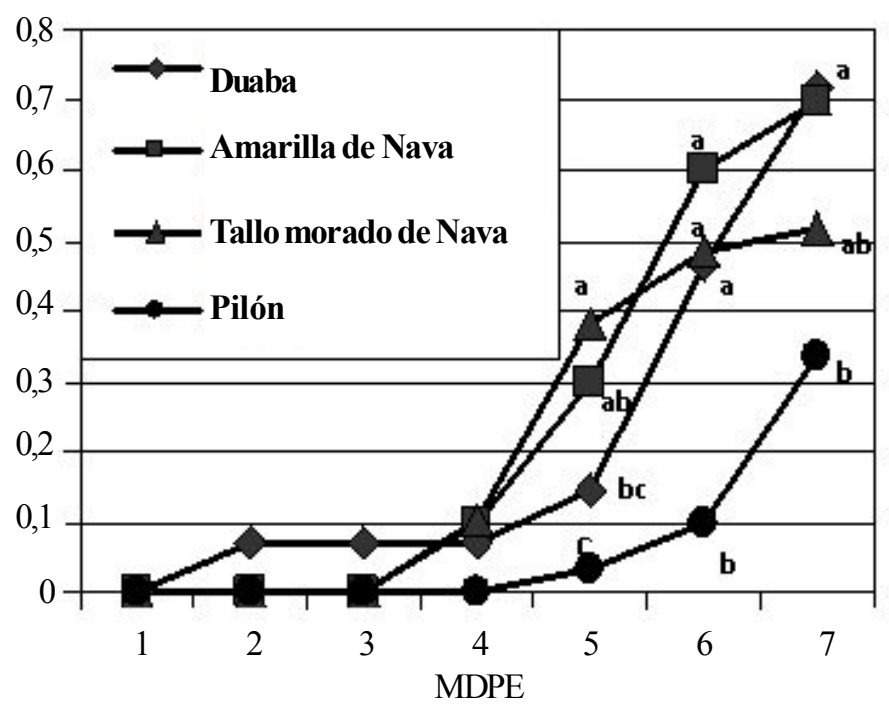

Figura 1. Incremento temporal de la proporción de plantas afectadas por PRSV-P de cuatro accesiones de papaya bajo condiciones de campo. *Letras distintas indicam difierencias estadisticas $(p \leq 0.05)$ entre las fuentes evaluadas en cada evaluación MDPE: meses después de la primera evaluación. 
proporción de 0.07 plantas enfermas a los cinco meses de plantadas (dos meses después de la primera evaluación). Esta proporción se mantuvo hasta los cinco meses después de la primera evaluación (MDPE) en que todas las accesiones mostraron plantas enfermas, aunque con proporciones que variaron entre ellas. En este momento, las accesiones de Tallo morado de Nava y Amarilla de Nava mostraron los mayores de 0.38 y 0.30 , respectivamente; que fueron estadísticamente superior ( $\mathrm{p} £ 0.05)$ a los alcanzados por las accesiones de Duaba (0.14) y Pilón (0.03).

A los seis MDPE la accesión de de Pilón mostró la menor proporción con un valor de 0.1 , que fue y inferior (p£0.05) al resto de las accesiones evaluadas. Al final del periodo evaluado (siete MDPE) las accesiones de Duaba y amarilla de Nava mostraron proporciones idénticas de plantas enfermas (0.7), mientras que las accesiones Tallo morado de Nava y Pilón mostraron proporciones inferiores ( $\mathrm{p} £ 0.05$ ) con valores de 0.5 y 0.3 , respectivamente. Siendo esta última la menos afectada por la enfermedad.

Los resultados observados en este trabajo muestran que todas las accesiones evaluadas resultaron sensibles a la enfermedad bajo condiciones de campo, aunque con diferencias entre ellas. Varias investigaciones desarrolladas en otros países sobre incidencia de plantas con PRSV a campo abierto han detectado altos niveles de infección por PRSV-P al cabo de los tres meses de plantados $(5,22,9,6)$. Incluso, en Taiwán, Bau et al. (7) detectaron plantas infectadas desde los 29 días posteriores a la siembra y ya al cabo de los cinco meses el $100 \%$ de las plantas se encontraron infectadas; y Rivas Valencia et al.

Tabla I. Intensidad de síntomas de mancha anular observados en tallo, pecíolo y follaje de selecciones criollas de papaya bajo condiciones de campo (ns. no significativo para $(\mathrm{p} \leq 0.05)$ ).

\begin{tabular}{lccc}
\hline \multirow{2}{*}{ Accessiones } & \multicolumn{3}{c}{ INTENSIDAD DE SíNTOMAS } \\
\cline { 2 - 4 } & Tallo & Peciolos & Follaje \\
\hline Tallo morado de Nava & 2,66 & 2,80 & 3,44 \\
Amarilla de Duaba & 2,23 & 3,03 & 4,03 \\
Amarilla de Nava & 1,90 & 2,41 & 3,78 \\
Sapote de Pilón & 1,70 & 2,21 & 3,61 \\
Coeficiente Variación & $29,72 \mathrm{~ns}$ & $19,69 \mathrm{~ns}$ & $12,119 \mathrm{~ns}$ \\
Error estándar & 0,219 & 0,149 & 0,13 \\
\hline
\end{tabular}

(19) detectaron plantas infectadas por el virus a los 47 días después del trasplante en experimentos con barreras de protección vegetal.

En estudios realizados por Rodríguez et al., (20) se determinó bajo condiciones de campo, que varios cultivares del grupo 'Solo' tuvieron un incremento más acelerado de plantas afectadas por PRSV$\mathrm{P}$, observándose plantas con síntomas a los tres meses después de la siembra en tres de cinco cultivares evaluados y proporciones finales entre 0.93 y 1.0. Resultados que coinciden con los observados por González y Rodríguez (13) en plantaciones de papaya cultivar Maradol Roja intercaladas con naranja Valencia (Citrus sinensis (L.) Osb.). Las diferencias existentes con el presente trabajo pudieran indicar una menor susceptibilidad de las accesiones a PRSV-P.

La Tabla I muestra la intensidad final de los síntomas de PRSV en tallo, pecíolo y follaje de las plantas evaluadas. No se observaron diferencias significativas ( $\mathrm{p} £ 0.05)$ entre las diferentes accesiones en ninguno de los órganos evaluados. Los síntomas en tallo mostraron valores de intensidad entre 1.7 (accesión de Pilón) y 2.66 para la accesión de tallo morado de Nava. Estos valores son bajos si se considera que en ninguna de las accesiones evaluadas se observaron síntomas de intensidad tres. Los síntomas observados en pecíolos mostraron mayor intensidad que los de tallo; en este caso los valores oscilaron entre 2.21 para la accesión de Pilón y 3.03 para la accesión criolla de Duaba.

Los síntomas foliares oscilaron entre 3.44 y 4.03 para las accesiones de tallo morado de Nava y Duaba, respectivamente. Estos fueron los órganos que mostraron mayor intensidad de la enfermedad, lo que sugiere su posible valor como diagnóstico de campo en programas de supresión de plantas enfermas. No obstante debe señalarse que en ninguno de los casos se observaron síntomas de intensidad superior a cuatro.

Bau et al. (7) encontraron síntomas severos de PRSV-P en el $100 \%$ de las plantas de una de las cuatro líneas de papayas transgénicas evaluadas bajo condiciones de campo en Taiwán, lo que indicó una alta susceptibilidad al virus. Sin embargo, los resultados observados en los diferentes órganos de las plantas evaluadas en nuestro trabajo, unido al progreso de la proporción de plantas enfermas, sugiere que nuestras accesiones son menos susceptibles a PRSV-P bajo condiciones de Cuba y que los cultivares del grupo 'Solo' introducidos en el país (20). Esta menor susceptibilidad pudiera atribuirse a una probable adaptación de estos cultivares a las razas de PRSV-P presentes en Cuba.

La Figura 2 muestra la intensidad de los síntomas de anillos

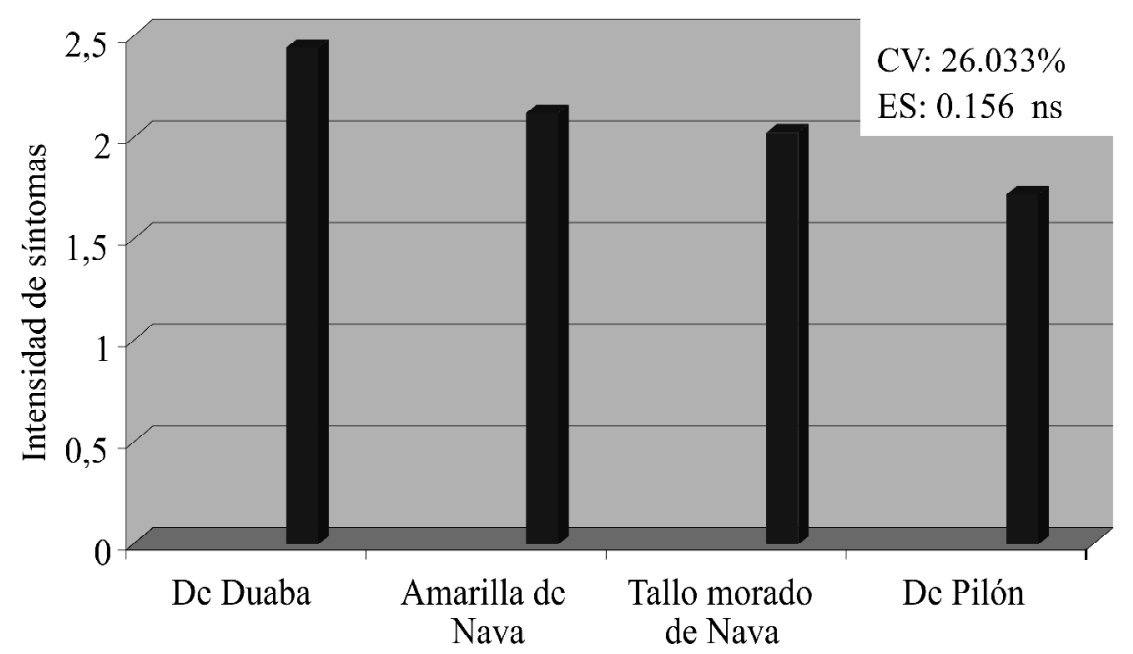

Figura 2. Intensidad de síntomas de mancha anular en la superficie de frutos de papaya de cuatro selecciones criollas evaluadas bajo condiciones de campo 
concéntricos de color verde oscuro observados en la superficie de los frutos de papaya en las diferentes accesiones evaluadas. En sentido general se observaron síntomas clasificados como leves de acuerdo a su intensidad y distribución en los frutos. Los valores no superaron el grado de 2.5 para ninguna de las accesiones, sin diferencias significativas entre ellas, pero con una tendencia a una menor intensidad para la accesión criolla de Pilón.

Los resultados de este trabajo respecto a los síntomas en frutos coinciden con los observados por Rodríguez et al. (20) en las accesiones Baixinho de Santa Amalia y BH-65 que mostraron intensidades inferiores a 2.5, mientras que los cultivares Sunset, Sunrise-Solo y Golden mostraron intensidades superiores a 3 según los resultados de estos mismos autores.

El hecho de la menor afectación de las accesiones evaluadas en el presente trabajo, sugieren una menor afectación de estos frutos y por consiguiente una posibilidad más amplia de comercialización como fruta fresca. A pesar de los resultados presentados en este trabajo, pudiera ser que en períodos más avanzados de la enfermedad sean observados otros síntomas como frutos de pequeño tamaño, ampollados y deformados (23). Se recomienda extender el período de evaluación de estos frutos bajo condiciones de campo, así como realizar análisis de calidad de los frutos de dichas accesiones, con el objetivo de cuantificar las afectaciones que pudieran existir en el aroma, sabor y composición química.

Este trabajo ofrece los primeros resultados sobre la susceptibilidad a PRSV-P de cuatro accesiones de papayo bajo condiciones de campo, poniendo una valiosa información en manos de los productores, así como para los programas de mejoramiento genético en el país.

\section{REFERENCIAS}

1. Aguilar-Ruiz, G.; Ávila-Quesada, G.; Barrios-Díaz, B., ChavarinPalacios, J.; Cristobal-Alejo, J.; González-Medina, R.; GutiérrezAyala, H.; Hernández-Martínez, M.; Higuera-Salazar, L.; JarabaNavas, J.; Misibaís-Loza, Y.; Mora-Aguilera, G.; Muñoz-Rodríguez, R.; Ramírez-Suarez, A.; Rivas-Valencia, P.; Romero, R.; Tovar, SA. Patrones espaciales de la incidencia de la mancha anular y su efecto en el tamaño y esquemas de muestreo. Congreso de la Sociedad Mexicana de Fitopatología, 26., Congreso Latinoamericano de Fitopatología, 10., 1999, Guadalajara, Memorias... Guadalajara: Editora, 1999, p. 286.

2. Alonso, M.; Tornet, Y.; Ramos, R.; Farrés, E; Rodríguez, D. Evaluación de dos híbridos de papaya introducidos en Cuba. Agronomía Costarricense, San José, v.33, n.2, p. 267-274, 2009.

3. Alonso, M.; Tornet, Y.; Ramos, R., Farrés; E., Aranguren; M.; Rodríguez, D. Caracterización y evaluación de dos híbridos de papaya en Cuba. Agricultura Técnica de México, Mexico City, v.34, n.3, p.333-339, 2008.

4. Alonso, M.; Tornet, Y.; Ramos, R.; Farrés, E.; Castro, J.; Rodríguez, M.C. Evaluación de tres cultivares de papaya del grupo solo basada en caracteres de crecimiento y productividad. Cultivos Tropicales, La Habana, v.29, n.2, p. 59-64, 2008.

5. Barbosa, F.; Pagnio, O. Papayo ring-spot virus: incidence and effect on yield of papaya (Carica papaya L.) (abstract). Review of Plant Pathology, Wallinford, v.63, p.2-3, 1982.

6. Bateson, M.F.; Lines, R.E.; Revill, P.; Chaleeprom, W.; Ha, C.V.; Gibbs, A.J.; Dale, J.L. On the evolution and molecular epidemiology of the potyvirus papaya ring-spot virus. Journal of General Virology, Reading, v.83, p.2575-2585, 2002.

7. Bau, H.J.; Cheng, Y.H.; Yu, T.A.; Yang, J.S.; Liou, P.C.; Hsiao,
C.H.; Lin, C.Y.; Yeh, S.D. Field evaluation of transgenic papaya lines carrying the coat protein gene of Papaya ringspot virus in Taiwan. Plant Disease, St. Paul, v.88, p.594-599, 2004.

8. Dantas, J.L.L. Introdução: mamão produção. In: Trindade, A.V. Mamão produção: aspectos técnicos. Brasília: Embrapa Mandioca e Fruticultura; Embrapa Comunicação para Transferência de Tecnologia, 2000. p. 5-13.

9. Espino de Paz, A.I.; Rodríguez Pastor, M.C.; Marrero Ferrer, M.; De León Rodríguez, J.M. Estudio preliminar sobre la incidencia y distribución de virosis en papaya (Carica papaya L.) en la isla de Gran Canaria. Phytoma, París, v.106, p.31-35, 1999.

10. Esquivel, M.A.; Bautista Alor, M.; Ortiz Garcia, M.; Moreno, A.Q.; Rohde, W.; Teyer, L.F.S. Characterising Cuban papaya accessions (Carica papaya L.) by AFLP markers. Revista Colombiana de Biotecnología, Bogotá, v.11, n.2, p.31-39, 2009.

11. Gonsalves, D. Papaya disease caused by viruses In: (Ed.) Plaetz, R.; Zentmyer, G.; Nishijima, W.; Rohrbch, K.; Ohr, H. Compendium of tropical fruit disease. APS Press, 1994, p. 96-98.

12. González, A.; Trujillo, G.; Vegas, A.; Garrido, M.J. Hospedantes de cepas del virus de la mancha anillada de la lechosa en Venezuela. Fitopatología Venezolana, Maracay, v.15, p.7-12, 1992.

13. González, L.; Rodríguez, D. Dinámica poblacional de vectores del virus de la mancha anular de la papaya intercalada con naranja Valencia y diseminación de la enfermedad. Centro Agrícola, Santa Clara, v.35, n.1, p.55-60, 2008.

14. Lima, R.C.A.; Lima, J.A.A.; Souza Jr., M.T.; Pio-ribeiro, G.; Andrade, G.P. Etiologia e estratégias de controle de viroses do mamoeiro no Brasil. Fitopatologia Brasileira, Brasilia, v.26, p.689702, 2001

15. Mora-Aguilera, G.; Nieto-Angel, D.; Téliz-Ortiz, D.; CampbellLee, C. Development of a prediction model for papaya ringspot in Veracruz, México. Plant Disease, St. Paul, v.77, p.1205$1211,1992$.

16. Pérez, M.C.; Correa, A.; Morera, S.; Ruiz, P. La industria citrícola cubana. Todo Citrus, Barcelona, v.15, p. 34-45, 2001.

17. Purcifull, D. Papaya ring-spot virus. Description of Plant Viruses, Warwick, n.84, 2 p., 1972.

18. Purcifull, D. Papaya ring-spot virus. Description of Plant Viruses, Warwick, n.292, 8 p., 1984.

19. Rivas-Valencia, P.; Mora-Aguilera, G.; Téliz-Ortiz, D.; MoraAguilera, A. Evaluation of plant barriers in an integrated management of papaya ringspot in Michoacan, Mexico. Summa Phytopathologica, Botucatu, v.34, n.4, p.307-312, 2008.

20. Rodríguez, D.; Tornet, Y.; Alonso, M.; Valero, L.; Peña, I.; Figueira, A.R.; Ramos, R. Severidade da mancha anelar do mamoeiro em diferentes genótipos do grupo Solo introduzidos em Cuba. Journal of Biotecnology and Biodiversity, Tocantins, v.2, n.4, p.28-36, 2011.

21. Tripathi, S.; Suzuki, J.Y.; Ferreira, S.A.; Gonsalves, D. Papaya ringspot virus-P: characteristics, pathogenicity, sequence variability and control. Molecular Plant Pathology, Oxford, v.9, n.3, p.269-280, 2008

22. Vegas, A.; Carmelí, M.; Trujillo, G. Importancia, distribución e incidencia de los virus que afectan a la lechosa (Carica papaya L.) en algunas zonas productoras del país. Agronomía Tropical, Maracay, v.14, p.213-237, 1985.

23. Vegas, A.; Trujillo, G.; Marys, E.; González, A.; Fermín, G.; Cermeli, M. El virus de la Mancha Anillada de la lechosa en Venezuela: descripción e importancia, medidas de prevención y control. Revista Digital CENIAP HOY, n.6, Maracay, Disponible en: $<$ www.ceniap.gov.ve/ceniaphoy/articulos/n6/arti/vegas_a/arti/ vegas_a.htm>. Fecha de consulta: 10 mar. 2005.

24. Yeh, S.D.; Gonsalves, D. Practices and perspectives of control of Papaya ringspot virus by cross protection. Advances in Disease Vector Research, New York, v.10, p.237-257, 1994. 